\title{
La divisibilidad del alma en la psicología de Aristóteles. ¿Es posible conciliar el hilemorfismo y el cardiocentrismo? ${ }^{1}$
}

\author{
César Augusto Mora Alonso ${ }^{2}$
}

Recibido: 22 de abril de 2017 / Aceptado: 15 de enero de 2018

Resumen. El propósito de este trabajo consiste en destacar el papel central que tiene el problema de la divisibilidad del alma en los dos enfoques bajo los que se presenta la investigación psicológica aristotélica: el hilemórfico y el cardiocéntrico. Mientras que el primero sostiene que el alma es la forma o esencia del cuerpo entero, el segundo aboga por la localización del alma en el corazón, pues asegura que allí se manifiestan los principios de las partes o facultades anímicas. A simple vista, podría suponerse que existe una incompatibilidad entre ambos enfoques, ya que no tendría mucho sentido afirmar que el alma se halla en todo el cuerpo y, al mismo tiempo, decir que se encuentra exclusivamente en uno de sus órganos específicos. Entonces, la tesis de este trabajo es que el pneuma ofrece la clave para la conciliación de estos dos enfoques, dado que es el instrumento empleado por las partes del alma para desplegar sus funciones por todo el cuerpo. El corazón, como sede material de dichas partes, irradia el pneuma en forma de sangre por todo el organismo; y éste, hasta que no se extingue esa operación vital, preserva su esencia, causa formal o razón de ser de su existir. De este modo, el hilemorfismo resulta sustentado por el cardiocentrismo.

Palabras clave: Alma, partes del alma, corazón, hilemorfismo, cardiocentrismo.

\section{[en] The divisibility of the soul in the Aristotle's Psychology. Is it possible to reconcile the Hylomorphism and the Cardiocentrism?}

\begin{abstract}
The purpose of this paper is to highlight the central role of the problem of divisibility of the soul in the two approaches under which the Aristotelian psychological research is presented: hylomorphism and cardiocentrism. While the first states that the soul is the form or essence of the whole body, the second advocates the location of the soul in the heart, because affirms that there are the principles of the parts of the soul. This could lead to assume that there is an incompatibility between the two approaches, since it would make no sense to say that the soul is in the whole body and, at the same time, declare that is found exclusively in one of its specific organs. For this reason, the thesis of this paper is that the pneuma provides the key to reconciling the two approaches, inasmuch as it is the instrument used by the parts of

1 Una versión previa de este texto fue presentada como ponencia en el VI Congreso Colombiano de Filosofía, realizado del 10 al 13 de agosto de 2016, en las instalaciones de la Universidad del Norte (Barranquilla, Colombia). Me gustaría agradecer a los profesores Marcelo Boeri y Henar Lanza por sus agudas observaciones, las cuales hicieron que repensara o confirmara mis intuiciones iniciales. Otra versión posterior también fue presentada como ponencia en el Congreso Internacional Aristóteles, 2400 años. La actualidad del pensamiento aristotélico. Evento celebrado en la Benemérita Universidad Autónoma de Puebla (Puebla, México) del 3 al 5 de octubre del mismo año. Estoy agradecido con Jean Orejarena y Jorge Quintana, miembros del comité organizador, por la invitación y deferencia. Buena parte de las ideas presentadas en este texto surgieron de las sesiones de estudio y discusión que mantuve con Jorge Quintana en la Universidad de Cartagena (Cartagena de Indias, Colombia). Así que mis respetos y amistad para él.

$2 \quad$ Universidad de Cartagena (Colombia). Programa de Filosofía. E-mail: cmoraa@unicartagena.edu.co
\end{abstract}


the soul to deploy its functions throughout the body. The heart, as a material seat of this parts, spreads the pneuma as blood throughout the body; and this, until no ends this vital function, preserves its essence, raison d'etre of its existence. Thus hylomorphism is supported by cardiocentrism.

Keywords: Soul, parts of the soul, heart, hylomorphism, cardiocentrism.

Sumario. 1. La importancia del problema de la divisibilidad del alma. 2. La solución del enfoque hilemórfico. 3. La solución del enfoque cardiocéntrico. 4. Una conciliación entre ambos enfoques.

Cómo citar: Mora Alonso, C. A. (2018) La divisibilidad del alma en la psicología de Aristóteles. ¿Es posible conciliar el hilemorfismo y el cardiocentrismo?, en Cuadernos de Filología Clásica. Estudios griegos e indoeuropeos 28, 129-139.

\section{La importancia del problema de la divisibilidad del alma}

En Ethica Nicomachea [EN] I, 13, 1102a5-1103a3, Aristóteles sostiene la necesidad de que el estudioso de la ética posea un conocimiento adecuado acerca del alma, dado que resulta fundamental para comprender por qué la felicidad es una actividad

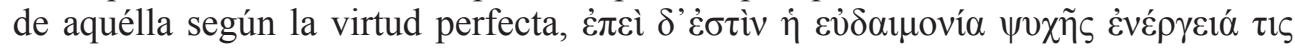

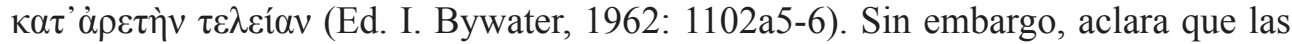
consideraciones respectivas deben ser preliminares, por cuanto se subordinan a la cuestión de cuáles son las condiciones indispensables para que el ser humano pueda alcanzar una vida buena.

Lo anterior lleva a que el filósofo de Estagira emplee un esquema general -al parecer de corte platónico- que divide al alma en dos partes principales: una ra-

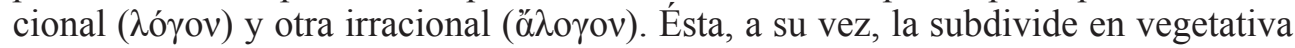
( $\varphi$ $\iota \kappa o ́ v)$, la cual es común a todos los seres vivos por ser el factor de la alimentación

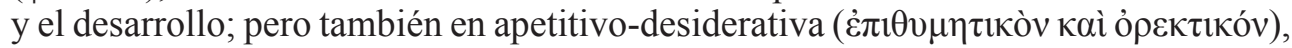
que se diferencia de la anterior por tener la capacidad de ser conducida por la parte racional del alma. Esta última, por lo demás, de acuerdo con EN VI, 1, 1139a3-15,

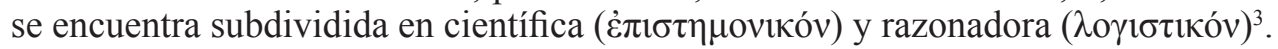

El hecho es que el Estagirita se vale de este esquema sólo para establecer a qué

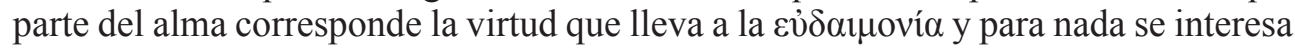
por examinar las implicaciones que tiene para el ámbito de la psicología. Por eso, en EN I, 13, 1102a28-32, afirma lo siguiente:

Nada importa para esta cuestión si éstas [las partes del alma $]^{4}$ se distinguen como las partes del cuerpo y todo lo divisible, o si son dos para la razón pero naturalmente inseparables, como en la circunferencia lo convexo y lo cóncavo (Trad. J. Pallí Bonet, 1998).

3 En términos generales, esta misma clasificación de las partes del alma aparece en Política [Pol.] (I, 5, 1254b2-9, 13, 1260a4-14, pero sobre todo en VII, 14, 1333a16-27 y 15, 1334b17-28). Básicamente, el contexto argumentativo es similar al de $E N$, pues está relacionado con lo que debería ser el papel de la educación para alcanzar las virtudes propias de cada quien. Aunque también lo que busca Aristóteles en Pol. I es subrayar la existencia por naturaleza de regentes y regidos. En su opinión, el amo debe ejercer su dominio sobre el esclavo como el alma lo hace sobre el cuerpo, lo racional sobre lo irracional y la inteligencia sobre el apetito.

4 Los corchetes y su contenido son míos. 


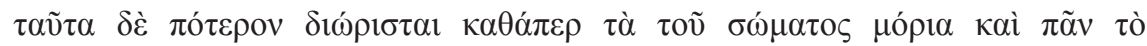

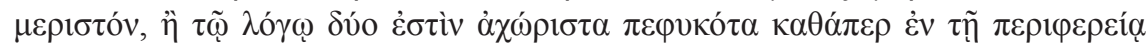

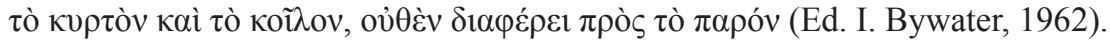

No obstante, en De Anima [de An.], Aristóteles sí le va a conceder un lugar privilegiado al problema de la divisibilidad de la $\psi v \chi \eta$, pues su resolución resulta decisiva para el propósito de este tratado, que es determinar con precisión lo que es el alma. Por ello, desde el principio, pregunta si puede ser divisible y, en caso de que lo sea, si la investigación debe comenzar por el alma en su conjunto, por cada una de sus partes constitutivas, por las operaciones que éstas ejecutan o, más bien, por los objetos sobre los que recaen dichas operaciones (I, 1, 402b1 y 9-16). De entrada, la advertencia es que el problema de las partes del alma está marcado por una dificultad capital, a saber:

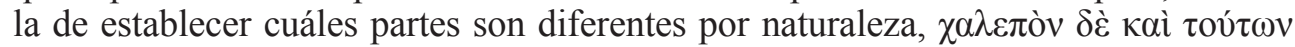

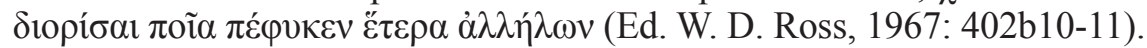

En efecto, en de An. III, 9, 432a19-432b7 y 10, 433b1-4, nuestro autor pone de relieve lo complicado que es hablar de partes del alma ( $\mu$ ópı $\alpha$ ז்̃ $\psi v \chi \tilde{\eta} \varsigma$ ), puesto que dan la impresión de ser múltiples, con el agravante de que no es tan claro diferenciarlas, porque pareciese que se encuentran íntimamente vinculadas; algo que se aprecia en las partes sensitiva ( $\alpha i \sigma \theta \eta \tau \imath \kappa o ́ v)$, imaginativa ( $\varphi \alpha v \tau \alpha \sigma \tau \imath \kappa o ́ v)$ y desiderati-

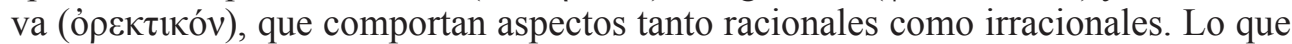
cuestiona la validez de las clasificaciones platónicas del alma, ya que la dividían sim-

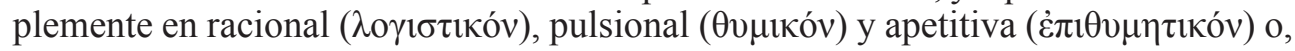

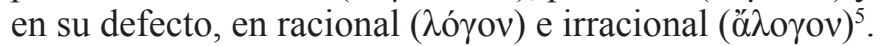

El filósofo de Estagira considera con más detenimiento el problema de las partes del alma en de An. I, 5, 411a26-411b30. Aquí expone una serie de interrogantes que apunta al hecho de si la ejecución de las diferentes operaciones anímicas -por ejemplo: vivir, percibir sensorialmente, etc.- corresponde al alma o a cada una de sus partes; esto último en caso de que sea divisible, claro está. Lo que lleva a indagar por aquello que la conserva unida, a pesar de que parezca segmentarse. En función de lo anterior, en 411b14-17, el Estagirita declara:

Cabría además preguntarse, en relación con las partes del alma, qué poder posee cada una de ellas respecto del cuerpo, ya que, si la totalidad del alma es la que mantiene unido a todo el cuerpo, conviene que, a su vez, cada una de ellas mantenga unida alguna parte del cuerpo (Trad. T. Calvo, 2003).

\footnotetext{
Esto significa que dichos esquemas carecen del rigor científico necesario para dar cuenta de «la verdadera naturaleza del principio vital». Ante todo, se debe tener presente que uno de los objetivos de Aristóteles es la confección de una taxonomía de todas las almas y poderes anímicos que ostentan los seres vivos (P. Gregoric, 2014: 8). En este orden de ideas, la tripartición del alma expuesta por Platón en República se impugna porque se funda en motivaciones netamente prácticas, pero sobre todo porque la ubicación de cada una de estas partes en regiones corporales distintas - cabeza, pecho y vientre, respectivamente- escinde al alma. Lo cual termina atentando contra lo que para Aristóteles sería una de sus funciones básicas, a saber: dotar de forma y unidad al cuerpo. Sin embargo, P. Vander Waerdt (1987: 641) anota que este esquema tripartito del alma le sirve al Estagirita de piedra de toque para establecer -en de An. II, 3, 414b2- los tres tipos de deseo (apetito, impulso y voluntad). Así como el bipartito sirve para los propósitos de EN y Pol. (1987: 628-632). En suma, según Vander Waerdt, ambos esquemas sólo se rechazan en el contexto científico de las obras biológicas aristotélicas (1987: 643). Una opinión parecida manifiesta W. Fortenbaugh (2002: 23-44). Quien explica la influencia platónica del esquema bipartito empleado por el Estagirita en sus obras ético-políticas y la razón por la cual no es incompatible con la división del alma presente en los tratados biológicos. P. Gregoric ofrece una guía detallada de esta cuestión en «Aristotle’s Philosophy of Mind» (2014: 1-23).
} 


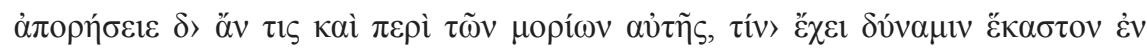

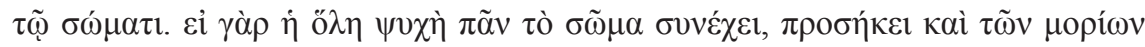

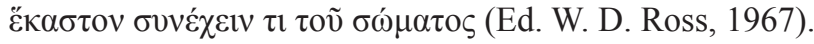

\section{La solución del enfoque hilemórfico}

En 411b26-27, luego de la alusión al fenómeno de la permanencia de la vida en los trozos de algunas plantas e insectos que han sido segmentados, se empieza a configurar una respuesta posible al problema planteado en la cita final del acápite anterior. La razón estriba en que se presenta el fraccionamiento del cuerpo, pero la conservación del alma en su totalidad en cada una de estas porciones corporales, «como si cada parte del alma no fuera separable de las demás, por más que el alma

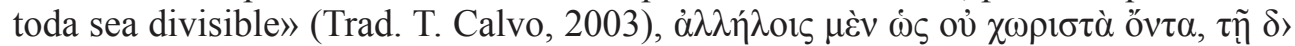

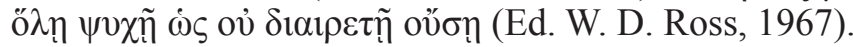

En de An. II, 2, 413b11-24, esto va a servir de piedra de toque para comenzar a asegurar que no resulta complicado establecer si una parte del alma puede ser divisible conceptualmente ( $\lambda$ ó $\gamma \omega)$ o espacialmente ( $\tau$ ó $\pi \omega)$. Una vez más, se advierte que hay plantas que siguen viviendo, a pesar de que se las haya dividido en partes. En este caso, Aristóteles viene a ratificar la impresión expresada en el párrafo anterior, pues pareciese que el alma que se encuentra en cada planta fuera una sola en entelequia,

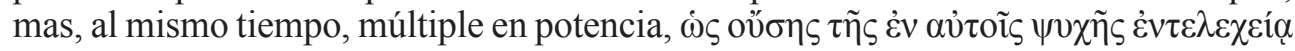

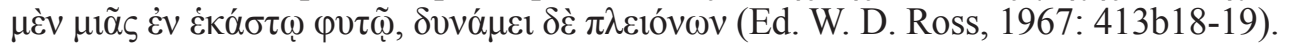

En este orden de ideas, también aduce que algo similar se presenta en el caso de aquellos insectos que han sido sometidos a un proceso de vivisección, por la razón de que cada uno de sus segmentos puede alcanzar a mantener diferentes facultades anímicas, verbigracia: la sensación ( $\alpha$ i̋ $\sigma \eta \eta \sigma ا \varsigma)$, el deseo (ő $\rho \varepsilon \xi \varsigma)$ y el movimiento lo-

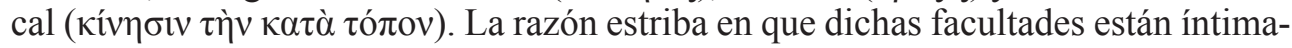
mente vinculadas, de manera que no resulta posible que se puedan dar separadas. En

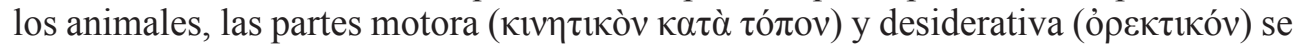
encuentran sujetas a las partes sensitiva ( $\alpha i \sigma \theta \eta \tau \iota \kappa o ́ v)$ y nutritiva $(\theta \rho \varepsilon \pi \tau \leftarrow \kappa o ́ v)^{6}$.

6 En otros pasajes de su obra biológica, el filósofo de Estagira también hace referencia al fenómeno de la permanencia de la vida en ciertos tipos de plantas e insectos que han sido cortados en partes. Una clara muestra de ello es De Partibus Animalium [PA] IV, 6, 682b28-33. Sin embargo, la diferencia entre ambos - de acuerdo con De Longitudine [Long.] 6, 467a19-30- está en que los insectos sólo pueden mantenerse vivos así por menos tiempo. La causa de esto obedece tanto a la ausencia de órganos como a la incapacidad de la facultad nutritiva para generarlos. Caso contrario al de las plantas, pues la facultad nutritiva presente en cada uno de sus trozos sí puede hacerlo, ya que cuentan con raíces y tallos en potencia. En De Incessu Animalium [IA] 7, 707a25-707b4, se afirma que las escolopendras y otros insectos alargados pueden albergar vida en cada uno de sus segmentos y, por ende, ejecutar los mismos movimientos que antes de ser divididos. Su constitución corporal es la que hace posible este fenómeno, dado que parecen estar hechos de modo análogo a un compuesto conformado por muchos animales. Algo así se afirma en PA IV, 5, 682a2-8. De Respiratione [Resp.] 3, 471b19-23 ubica en este grupo a los ciempiés. Historia Animalium [HA] IV, 7, 531b30-532a5 expresa una opinión similar. Mas también dice que los insectos que tienen una constitución muy fría, o que se enfrían muy pronto debido a su contextura diminuta, no pueden sobrevivir cuando han sido divididos. $I A 7,707 \mathrm{a} 25$ descarta esta posibilidad también para los animales sanguíneos. Aunque las tortugas serían la excepción por tener una estructura parecida a la de los insectos, según De Inventute [Iuu.] 2, $468 \mathrm{~b} 15$ y Resp. 17, 479a3-7. Intérpretes como R. K. Sprague (1989: 3436), D. Lefebvre (2002: 23-28), A. P. Bos (2007: 96-98) y J. Mittelmann (2011: 84-87) han tratado estos problemas. Todos ellos aportan valiosos análisis sobre las evidencias empíricas que Aristóteles utiliza para rebatir la separación espacial de las partes del alma. 
Para Aristóteles, lo anterior puede comprenderse mejor si se aporta una definición rigurosa de la $\psi v \chi \eta ́$. De acuerdo con de An. II, 1-4, ésta es el principio esencial que actualiza o le imprime vida a un cuerpo natural capacitado para tenerla. Lo que implica que el alma no sea un cuerpo, dado que no es materia, sino algo del cuerpo, su causa vital. Aquella que garantiza el correcto funcionamiento de los órganos corporales, que son los vehículos de expresión de las distintas partes o facultades anímicas. Según este tratado psicológico, las susodichas son las siguientes: nutritiva, sensiti-

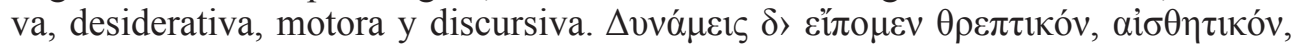

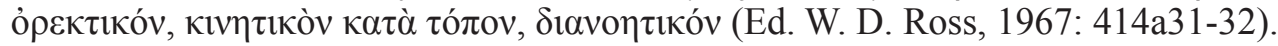

Con esta concepción, nuestro autor se aproxima a una respuesta más precisa al problema de la divisibilidad. En de An. II, 1, 413a3-6, él asegura:

Es perfectamente claro que el alma no es separable del cuerpo o, al menos, ciertas partes de la misma si es que es por naturaleza divisible: en efecto, la entelequia de ciertas partes del alma pertenece a las partes mismas del cuerpo (Trad. T. Calvo, 2003).

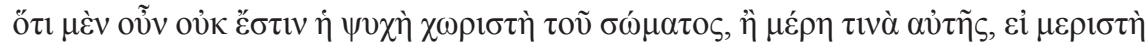

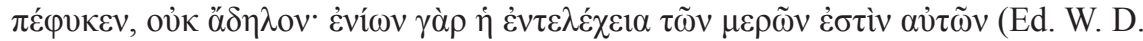
Ross, 1967).

En síntesis, la causa por la cual el alma no puede ser desligada del cuerpo -y de que sus partes no puedan distinguirse de modo tangible, como cuando se diferencia a un brazo de una pierna- radica en que no es un ente concreto, un compuesto de

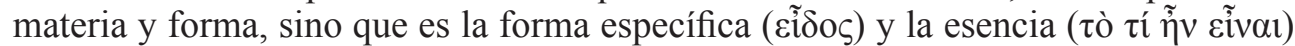
de un cuerpo.

Pero aquí no se acaba la cuestión. En de An. II, 2-3, el filósofo de Estagira sostiene que la definición del alma sólo es posible gracias a sus partes o facultades, a pesar de que aquélla sea el principio de éstas. Dichas capacidades anímicas aparecen en los seres vivos de manera escalonada, de tal modo que las posteriores no puedan darse sin la presencia de las previas ${ }^{7}$. Lo anterior servirá de piedra de toque para desarrollar la explicación sobre la divisibilidad del alma en de An. II, 2, 413b11-32. El argumento va a consistir en que la $\psi v \chi \eta ́$ no puede ser segmentada en partes concretas, sino sólo lógicamente o por medio de la definición. Esto es lo que se expresa al final del pasaje (27-32):

En cuanto al resto de las partes del alma se deduce claramente de lo anterior que no se dan separadas como algunos pretenden. Que son distintas desde el punto de vista de la definición es, no obstante, evidente: la esencia de la facultad de sentir difiere de la esencia de la facultad de opinar de igual manera que difiere el sentir y el opinar; y lo mismo cada una de las demás facultades mencionadas. (Trad. T. Calvo, 2003).

\footnotetext{
La tesis del escalonamiento de las facultades anímicas se expone en varios pasajes de de An. II, 2 (413a31413b13, 413b32-414a3) y 3 (414a29-415a13). Básicamente, consiste en la distribución sistemática de una o más capacidades de las que dispone un animal a partir de otra que le sirve de punto de partida. Así las cosas, la nutritiva es condición de existencia de la sensitiva. Entre tanto, la desiderativa parte de esta última y es determinante para el surgimiento de la motora. Por lo demás, quienes poseen la facultad discursiva poseen necesariamente todas las restantes. Para esta tesis es claro que una facultad posterior presupone a una anterior, pero no a la inversa.
} 


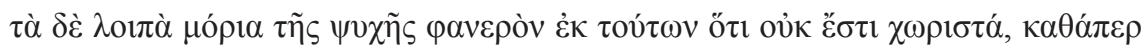

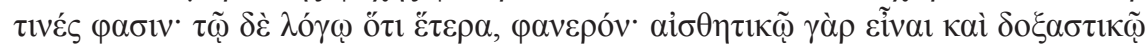

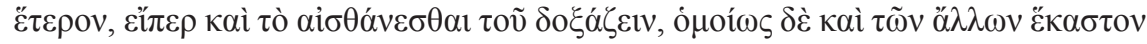

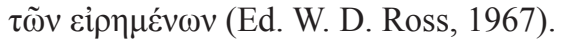

La consecuencia de esta argumentación es que alma y cuerpo constituyen un compuesto indisoluble llamado ser viviente. El alma es la forma que actualiza y le da vida a lo corporal. A su vez, las partes o facultades del alma son las capacidades para ejecutar las diferentes operaciones vitales, mientras que el cuerpo y sus órganos respectivos son los instrumentos de ejecución. En opinión del filósofo de Estagira, la facultad nutritiva es la más básica de todas, debido a que es la responsable de la preservación -tanto en plantas como en animales- del calor vital ( $\theta \varepsilon \rho \mu$ ó $\eta \tau \tau \alpha)$, condición que diferencia a lo animado de lo inanimado, y que por ende posibilita los procesos de alimentación y reproducción (de An. II, 4, 416a19-416b31).

\section{La solución del enfoque cardiocéntrico}

De acuerdo con de An. II, 2, 413b1-10, lo que distingue a los animales de las plantas es la posesión de la facultad sensitiva, de la que el tacto es su modo primario. En otros pasajes de esa misma obra -por ejemplo: II, 11, 423b22-31, III, 12, 434b18-24 y 13, 435b2-19- Aristóteles pone de manifiesto que el tacto y el gusto, que es una especie de aquel sentido, son vitales para la existencia del animal, pues se encuentran íntimamente vinculados con la parte nutritiva del alma. De igual manera, asegura que el órgano que percibe las cualidades tangibles (caliente-frío, seco-húmedo, duroblando) es el táctil, el cual es una parte interna del cuerpo. Asimismo, lo que dice en II, 8, 420b22-29 resulta significativo, por cuanto da a entender que ciertas partes del alma se alojan en partes determinadas del cuerpo, verbigracia: en los órganos responsables de la respiración y de la voz, a saber: laringe, tráquea, pulmón y corazón.

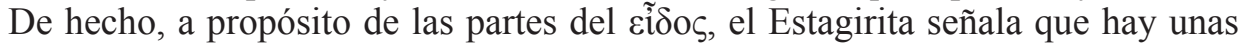
partes del animal tan necesarias que sin ellas no podría tener lugar la vida, o sea, el alma. Por lo menos, así lo plantea en Metaphysica [Metaph.] Z, 10, 1035b25-27:

Y algunas son simultáneas, y en tal caso están las indispensables y sin las cuales no puede darse el concepto ni la substancia; por ejemplo, suponiendo que sea tal, el corazón o el cerebro; pues nada importa que sea lo uno u otro (Trad. V. García Yebra, 1998).

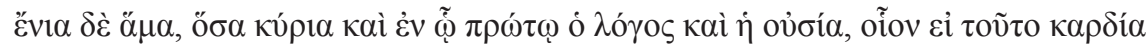

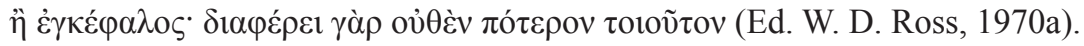

El filósofo de Estagira arroja algunas luces sobre esta cuestión en De Sensu [Sens.] 2, 438b25-439a5, ya que indica que el cerebro es la parte más húmeda y fría del cuerpo, a diferencia del corazón, que es la más caliente. A su juicio, esta sería la causa de que los órganos encargados de la nutrición - es decir, los de gusto y tacto- se hallen cerca de la región cardiaca. Ahora bien, este punto de vista encaja perfectamente con lo expuesto en de An. por varias razones: en primer lugar, porque aquí se afirma que los procesos digestivos que conservan la vida del animal se dan gracias a 
la presencia del calor vital; en segundo lugar, dado que se sostiene que los animales terrestres tiene más calor que el resto debido a la tenencia de pulmones; $\mathrm{y}$, en tercer lugar, por el hecho de que es la región susodicha la que primordialmente requiere de la respiración (de An. II, 4, 416b28-29 y 8, 420b22-26).

No en vano, Aristóteles advierte que en el tronco $(\theta \omega \rho \alpha \xi)$ se concentra el núcleo de la actividad vital, pues ahí se encuentran los órganos corporales más importantes. Esto le va a servir de piedra de toque para sustentar que el alma como principio vital incorpóreo reside en lo somático, fundamentalmente en el corazón, la parte con mayor poder (Iuu.1, 467b13-16). La causa radica en que dicho órgano es el encargado de mantener unido al cuerpo por ser la fuente de calor que mantiene con vida al animal. De ahí que llegue a expresar que el alma pareciese estar como ardiendo en

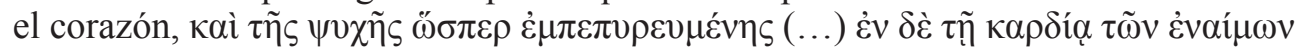
(Ed. W. D. Ross, 1970b: 4, 469b16-17). Un argumento contundente para considerarlo -de acuerdo con $P A$ III, 4, 666a22 y 666b17-como una especie de ser viviente

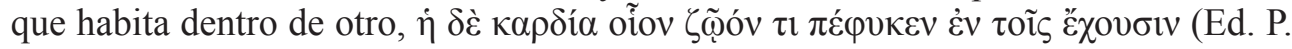
Louis, 1956).

En efecto, el corazón es lo primero que se forma en embriones y fetos. En este sentido, se presenta como el principio constitutivo de los animales sanguíneos, puesto que es el responsable de su crecimiento y desarrollo. A partir de él, se originan la sangre, las venas y las partes corporales homogéneas y heterogéneas (De Generatione Animalium [GA] II, 4, 740a1-19). Igualmente, cumple un papel determinante en la alimentación y conservación del animal, por la razón de que ejecuta la transformación del alimento en sangre para distribuirla por todo el organismo a través del aparato circulatorio (De Somno [Somn.Vig.] 2, 456b1-5). De esta manera, el corazón se revela como la base material de la facultad nutritiva.

Sin embargo, la к $\alpha \rho \delta i ́ \alpha$ también funge como sede material de las facultades anímicas restantes. Según Iuu. 3, 469a11-14, en el órgano en cuestión reside el principio de la facultad sensitiva, dado que allí convergen los cinco sentidos. Por ello, el filó-

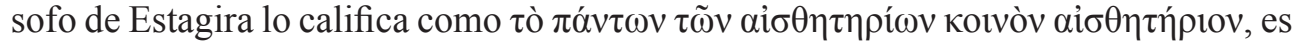
decir, como el órgano sensorial que se encarga de mantener vinculados a los demás.

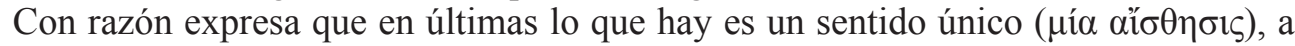
pesar de la existencia de los distintos tipos de sensación. De acuerdo con Somn.Vig. 2, 455a21-22, el corazón sería el órgano sensorial soberano (

De otro lado, el corazón es principio de lo desiderativo por ser punto de partida y de llegada de los movimientos provocados por el placer y el dolor ( $P A$ III, 4, 666a1113), puesto que cuando se experimenta un deseo se produce una agitación en el corazón como resultado de la ebullición de la sangre o del elemento caliente que lo circunda (de An. I, 1, 403a31-403b1, 4, 408b7-8 y III, 9, 432b31-433a1).

Por su parte, la abundancia de tendones es una de las razones por las cuales Aristóteles considera que el corazón es el principio de la facultad motora, ya que los tendones le sirven de medio para desplegar toda su fuerza y trasmitirla a las articulaciones ( $P A$ III, 4, 666b14-17). Lo que significa que viene a ser el primer responsable de la contracción y distención de los músculos del cuerpo. Algo parecido sostiene en De Motu Animalium [MA] 9, 702b20-25, pues explica que el movimiento tiene su origen en la alteración de la zona que circunda a lo cardiaco. Dicha alteración produce una afección de las partes más próximas, las que a su vez terminan por modificar a las extendidas y contraídas. Por eso, en las líneas inmediatamente posteriores (2628), afirma que el órgano de la $\kappa \alpha \rho \delta i ́ \alpha$ cumple múltiples funciones al mismo tiempo: 
(...) pues los miembros se mueven simultáneamente a partir de un principio, y cuando uno permanece quieto, el otro se mueve (Trad. A. Alonso Miguel, 2000).

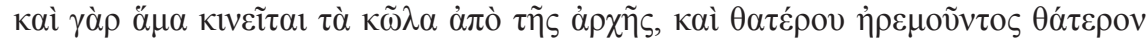

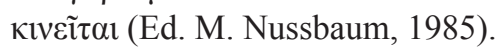

De igual manera, hasta la facultad discursiva requiere del concurso del corazón. de An. I, 4, 408b24-25, por ejemplo, pone de relieve que el inteligir y el contemplar se debilitan a partir del deterioro de ciertos órganos. Uno de ellos sería el cardiaco, si es que el discurrir consiste en que el corazón se mueva de cierto modo (408b8-9). De Memoria [Mem.] 2, 453a14-453b7 arroja más luces sobre la relación entre la facultad discursiva y el órgano mencionado. Aquí se deja claro que la reminiscen-

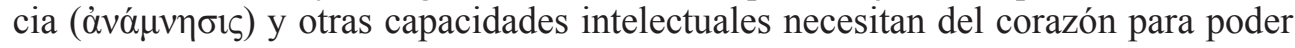
desempeñar sus funciones. La causa estriba en que las imágenes y los recuerdos -insumos a partir de los cuales se elaboran los conceptos- son estados o afecciones de la $\kappa \alpha \rho \delta i ́ \alpha$. De suerte que esta parte corporal resulta ser determinante incluso para el advenimiento del conocimiento intelectual ${ }^{8}$.

\section{Una conciliación entre ambos enfoques}

En virtud de todo lo expuesto, podrían tenerse como contradictorios los planteamientos de la psicología del Estagirita, dado que son dos los enfoques que ha empleado en su indagación en torno al alma, a saber: el hilemórfico y el cardiocéntrico. El primero sostiene que el alma es la forma o esencia del cuerpo entero. Éste se mantiene vivo gracias a la presencia de aquélla en todas y cada una de sus partes constitutivas. El segundo, entre tanto, asegura que el corazón es la sede del alma, por la razón de que en dicho órgano está el principio de las facultades nutritiva, sensitiva, desiderativa y motora. A simple vista, estos dos enfoques muestran su incompatibilidad, pues parecería no tener mucho sentido afirmar que el alma se encuentra en todo el cuerpo y al mismo tiempo decir que se localiza exclusivamente en una de sus partes específicas.

Ahora bien, K. Corcilius y P. Gregoric (2013: 87-89) aportan elementos de juicio para superar esta dificultad. De acuerdo con ellos, la clave radica en comprender que en de An. Aristóteles ofrece en líneas generales su doctrina psicológica, la cual postula que los seres vivos son un compuesto de alma y cuerpo. Mientras que en los tratados biológicos profundiza en el estudio de los procesos psicofísicos que experimentan tales seres, al interesarse por cuestiones como la corporeidad del alma y el desarrollo del cardiocentrismo que, en la práctica, implica la adaptación anatómica y fisiológica del hilemorfismo.

De hecho, la propuesta de este trabajo se inscribe en la línea interpretativa de K. Corcilius y P. Gregoric, puesto que busca rastrear y profundizar en las conexiones

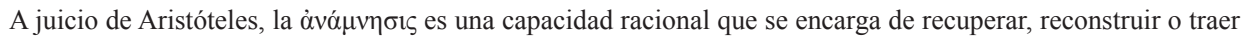
a colación las diferentes clases de recuerdos que han sido olvidados, a saber: imágenes, experiencias e intelecciones (Mem. 2, 451b2-5). Se trata de una capacidad que ejecuta operaciones dianoéticas complejas, tales como indagaciones, asociaciones e inferencias. En este sentido, la ỏvó la hora de calcular y deliberar (Mem. 2, 453a9-14, de An. III, 10, 433b29 y 11, 434a5-10). Empero, lo llamativo de este asunto es que Aristóteles sostiene abiertamente que en el despliegue de esta capacidad interviene el funcionamiento del sistema cardiovascular (Mem. 2, 453a21-23).
} 
que pueden mantener estos dos enfoques de la psicología aristotélica. En este orden de ideas, de An. II, 12, 424a24-424b3, desempeña un papel crucial en este cometido, ya que enuncia que la facultad sensitiva -pese a que se halla en el órgano primario ( $\alpha i \sigma \theta \eta \tau \eta ́ \rho ı v ~ \pi \rho \tilde{\omega} \tau o v)$ o corazón- se distingue esencialmente de éste, en la medida

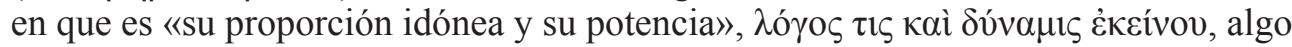
muy distinto de la mera magnitud o extensión. Lo que viene a significar que ese $\lambda$ ó ${ }_{0}$ y esa $\delta$ v́ $\alpha \mu \iota \varsigma$ constituyen el principio que informa e imprime fuerza a la parte central del organismo.

El Estagirita expresa esta clave conciliatoria a partir de $M A 10,703$ a18-19, dado que allí esclarece la relación entre alma, soplo innato $(\pi v \varepsilon \tilde{u} \mu \alpha)$ y corazón. Dicho $\pi v \varepsilon \tilde{v} \mu \alpha$ es -como lo pone de relieve A. P. Bos (2003: 31-46)- el instrumento que el alma emplea para actuar en el cuerpo, pues «está claro que por naturaleza puede ser

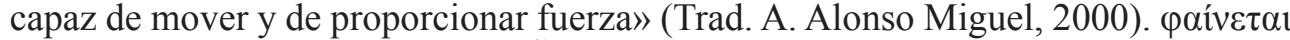

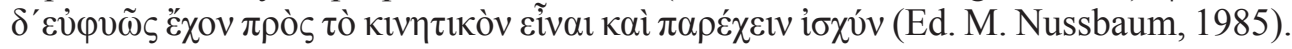
Esta razón hace que Aristóteles le asigne como sede el principio del sistema cardiovascular, porque es el lugar en el que las facultades del alma despliegan, a través de este soplo innato, sus funciones por todo el cuerpo. Lo anterior le va a permitir comparar al animal con una ciudad bien gobernada en 10, 703a28-703b2:

Así pues, se ha dicho con qué parte, al ser movida, el alma mueve, y la causa de ello; pero hay que suponer que el animal está constituido como una ciudad bien gobernada. En efecto, en la ciudad, una vez que el ordenamiento queda establecido, no hay en absoluto necesidad de un monarca diferente que deba estar presente en cada uno de los acontecimientos, sino que cada uno por sí mismo hace lo que le corresponde según está establecido, y tal cosa sigue a tal otra en virtud de la costumbre; en los animales esto mismo ocurre en virtud de la naturaleza, es decir, que cada una de las partes que los constituyen realiza su trabajo por ser así por naturaleza, de manera que en cada una no hace falta que haya ningún alma, sino que, al estar ésta en el principio del cuerpo, las otras partes viven por estar naturalmente unidas a él, y realizan por naturaleza el trabajo que les es propio (Trad. A. Alonso Miguel, 2000).

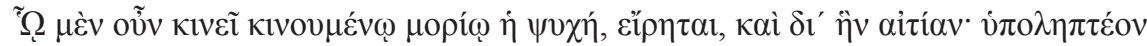

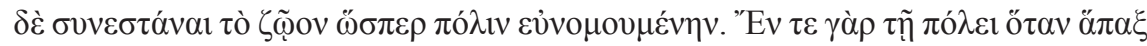

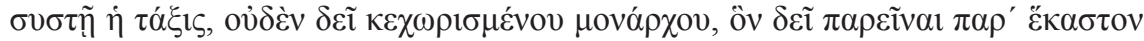

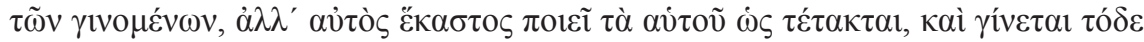

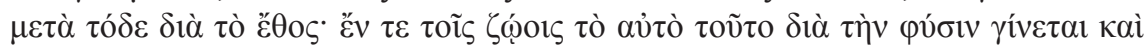

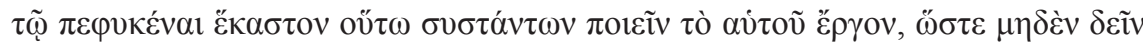

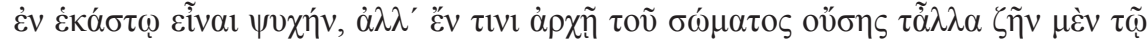

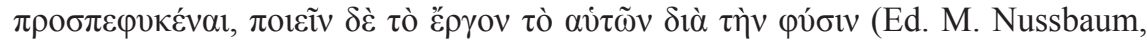
1985).

En suma, la tesis de este trabajo es que para el filósofo de Estagira tiene sentido hablar de la divisibilidad del alma en partes, pero sólo desde el punto de vista conceptual, pues el ser vivo, en cuanto compuesto de alma y cuerpo, constituye un todo indisoluble. Algo en lo que concuerdan tanto hilemorfismo como cardiocentrismo, lo que, a su vez, posibilita la conciliación entre estos dos enfoques aparentemente in- 
compatibles de la psicología aristotélica. El corazón -sede material de las facultades anímicas- irradia el $\pi v \varepsilon \tilde{u} \mu \alpha$ en forma de sangre por todo el organismo; y éste, hasta que no se extingue esa operación vital, preserva su esencia, causa formal o razón de ser de su existir. Sin embargo, habrá que ver cómo encaja en este planteamiento el voũ $($ (intelecto) del alma humana, ya que en diversos pasajes del Corpus Aristotelicum se insiste en que es divino, inmaterial e incorruptible. Motivos de peso para no atribuirle un órgano corporal ${ }^{9}$.

\section{Referencias bibliográficas}

\section{Ediciones y traducciones de la obra aristotélica}

Alonso Miguel, A. (2000), Marcha de los animales [IA]. Movimiento de los animales [MA]. Madrid. Editorial Gredos. Introducción, traducción y notas.

Bernabé Pajares, A. (1987), Tratados breves de historia natural. Madrid. Editorial Gredos. Introducción, traducción y notas.

Bywater, I. (1962), Aristotelis ethica Nicomachea [EN]. Oxford. Clarendon Press. Texto griego, introducción y comentarios.

Calvo, T. (2003), Acerca del alma [de An.]. Madrid. Editorial Gredos. Introducción, traducción y notas.

Drossaart Lulofs, H. J. (1972), De Generatione Animalium [GA]. Oxford. Clarendon Press. Texto griego, introducción y comentarios.

Forster, E. S. (1961), Progression of Animals (De Incessu Animalium) [IA]. Cambridge. The Loeb Classical Library. Texto griego con traducción al inglés.

García Valdés, M. (1988), Política [Pol.]. Madrid. Editorial Gredos. Introducción, traducción y notas.

García Yebra, V. (1998), Metafísica de Aristóteles [Metaph.]. Madrid. Editorial Gredos. Edición trilingüe (greco-latino-española).

Jiménez, E. (2000), Partes de los animales [PA]. Madrid. Editorial Gredos. Introducción, traducción y notas.

Louis, P. (1956), Les parties des animaux (De Partibus Animalium) [PA]. París. Les Belles Lettres. Texto griego con traducción al francés, introducción y comentarios.

- (1964-1969), Historie des animaux (Historia Animalium) [HA]. París. Les Belles Lettres. Texto griego con traducción al francés, introducción y comentarios.

Nussbaum, M. (1985), Aristotle's De Motu Animalium [MA]. Princeton. Princeton University Press. Texto griego con traducción al inglés, comentarios y ensayos interpretativos.

Pallí Bonet, J. (1992), Investigación sobre los animales [HA]. Madrid. Editorial Gredos. Introducción, traducción y notas.

- (1998), Ética Nicomáquea [EN]. Madrid. Editorial Gredos. Introducción, traducción y notas.

Ross, W. D. (1964), Politica [Pol.]. Oxford. Clarendon Press. Texto griego, introducción y comentarios.

9 En efecto, en de An. III, 4, 429a24-27, en relación con el intelecto, se plantea lo siguiente: «De ahí que sería igualmente ilógico que estuviera mezclado con el cuerpo: y es que en tal caso poseería alguna cualidad, sería frío o caliente y tendría un órgano como lo tiene la facultad sensitiva; pero no lo tiene realmente» (Trad. T. Calvo,

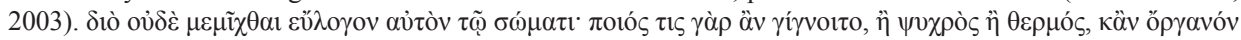

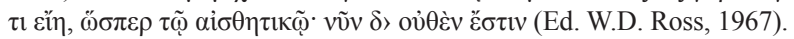


— (1967), De Anima [de An.]. Oxford. Clarendon Press. Texto griego, introducción y comentarios.

— (1970a), Aristotle's Metaphysics. [Metaph.]. 2 Vols. Oxford. Clarendon Press. Texto griego, introducción y comentarios.

- (1970b), Parva Naturalia. Oxford. Clarendon Press. Texto griego, introducción y comentarios.

Sánchez, E. (1994), Reproducción de los animales [GA]. Madrid. Editorial Gredos. Introducción, traducción y notas.

\section{Literatura crítica}

Bos, A. P. (2003), The soul and its instrumental body. Amsterdam. Brill.

- (2007), «Aristotle on dissection of plants and animals and his concept of the instrumental soul-body», AncPhil, 27: 95-106.

Corcilius, K., y Gregoric, P. (2013), «Aristotle's Model of Animal Motion», Phronesis, 58: 52-97.

Fortenbaugh, W. (2002), Aristotle on emotion. London. Duckworth.

Gregoric, P. (2014), «Aristotle's Philosophy of Mind», en D. Clayman (ed.), Oxford Bibliographies in Classics, New York: 1-23. DOI: 10.1093/OBO/9780195389661-0174.

Lefebvre, D. (2002), «L'argument du sectionnement des vivants dans les Parva Naturalia: Le cas des insects", RPhA, 20: 5-34.

Mittelmann, J. (2011), «A Bug's Life. Aristotle's Metaphysic of Divided Insects», The New Centennial Review, 10 (3): 79-104.

Sprague, R. K. (1989), «Aristotle and Divided Insects», Méthexis, 2: 29-40.

Vander Waerdt, P. A. (1987), «Aristotle's Criticism of Soul-Division», AJPh, 108 (104): $627-$ 643. 